\title{
EXPERIENCIAS Y NECESIDADES DE OCIO DE LOS JÓVENES CON DISCAPACIDAD. UN ESTUDIO EXPLORATORIO EN EL PAÍS VASCO (ESPAÑA)
}

\author{
LEISURE EXPERIENCES AND NEEDS OF YOUNG PEOPLE WITH DISABILITIES. \\ AN EXPLORATORY STUDY IN THE BASQUE COUNTRY (SPAIN)
}

\author{
Aurora Madariaga Ortuzar ${ }^{1}$ \\ Idurre Lazcano Quintana ${ }^{2}$
}

\section{Resumen}

Este artículo presenta los resultados de una investigación de carácter integral "El ocio de la juventud con discapacidad" que se fundamenta en cuatro ideas fuerza: el ocio desde una visión humanista como derecho y experiencia; la concepción de discapacidad; la juventud con discapacidad como objeto de estudio, y la inclusión como herramienta de intervención desde un enfoque estratégico inclusivo de la intervención en diferentes ámbitos del ocio. En este trabajo está presente la necesidad de desarrollar estrategias de ocio inclusivo que permitan avanzar con relación a la promoción de experiencias y vivencias satisfactorias de ocio y a la participación de la juventud con discapacidad en la oferta de ocio comunitaria. Los resultados centrados en la demanda muestran que la juventud con discapacidad, al igual que el resto de la población joven, valora el ocio como elemento importante en sus vidas, como un espacio vital adecuado para fraguar nuevas amistades, y como ámbito de desarrollo personal. Con relación a la oferta de ocio comunitaria, pública o privada, esta no siempre garantiza el acceso y la participación de la juventud con discapacidad porque no cumple las condiciones de accesibilidad o no incluye medidas que faciliten su participación.

Palabras claves: juventud; discapacidad; experiencia de ocio; demandas en ocio; participación; accesibilidad en la oferta de ocio; inclusión social

\section{Abstract}

This article presents the results of an investigation about "Leisure of young people with disabilities" which is based on four key ideas: leisure from a humanistic vision as a human right and an integral experience; the concept of disability; young people with disabilities as objects of intervention, and inclusion as a tool of intervention. This paper is concerned with the need for developing strategies for inclusive leisure that fosters satisfactory experiences, leisure moments and also the participation of youth with disabilities in the leisure offer of the community. The results, which are focused on the demand for these spaces, show that young people with disabilities, like any other young person, value leisure as an important element in their lives, as a suitable environment to forge new friendships, and as an area for personal development. The communal leisure, whether public or private, does not always guarantee access and participation of young people with disabilities because they do not have the necessary accessibility conditions or do not include strategies to ease their participation.

Keywords: youth; disability; leisure experience; demands leisure; participation; accessibility in leisure,; social inclusion

Fecha de recepción: 24 de octubre de 2015

Fecha de aprobación: 8 de marzo de 2016

Para citar este artículo:

Madariaga, A. y Lazcano, I. (2016). Experiencias y necesidades en ocio de los jóvenes con discapacidad. Un estudio exploratorio en el País Vasco (España). Lúdica Pedagógica, (23), 71-83.

1 Doctora en Ocio y Potencial Humano. Máster en Educación Especial. Experta en Pedagogía Terapéutica. Licenciada en Psicología. Instituto de Estudios de Ocio. Universidad de Deusto. Correo electrónico: aurora.madariaga@deusto.es

2 Doctora en Ocio y Potencial Humano. Máster en Dirección de Proyectos de Ocio. Licenciada en Pedagogía. Instituto de Estudios de Ocio. Universidad de Deusto. Mail: ilazkano@deusto.es 


\section{CONTEXTO DEL ESTUDIO}

España es posiblemente el país de Europa con una mayor tradición en estudios sobre juventud, existen multitud de investigaciones que analizan la realidad del ocio de las personas jóvenes, aunque no son tan abundantes las centradas en el colectivo de jóvenes con discapacidad (Doistua, Lazcano y Madariaga, 2011; Madariaga y Lazcano, 2014).

El estudio (Madariaga, Lazcano y Doistua, 2010) desarrolla un tema que tiene un gran interés por varias razones: la importancia del ocio en la sociedad actual (Cuenca, 2000), los paradigmas vigentes relacionados con la discapacidad, el conocimiento del ocio de la juventud con discapacidad que vive y madura en una sociedad del siglo xxI y la aplicación de la inclusión al ocio. Desde el inicio se trabajó activamente con toda la red de agentes implicados que trabajan en ocio, juventud y/o discapacidad.

El proyecto, de carácter integral (San Salvador del Valle y Lazcano, 2003), incluye cuatro partes: el desarrollo conceptual y la recogida de estadísticas, el estudio de las demandas de la juventud con discapacidad (Lazcano, Doistua, Lázaro y Madariaga, 2012), el análisis de la oferta comunitaria de ocio y oferta asociativa del ámbito del ocio y la discapacidad, y el diseño de una estrategia de inclusión en ocio (Cátedra Ocio y Discapacidad, 2014). Este artículo se centra en la experiencia de ocio de la juventud con discapacidad y sus necesidades en el acceso a la oferta de ocio comunitaria. La metodología empleada en el estudio es variada y diversa y se ajusta a los objetivos planteados en cada parte.

Las diferentes herramientas (Madariaga y Lazcano, 2014) han sido diseñadas, revisadas, y contrastadas para su utilización en esta investigación. Para analizar la experiencia de ocio se realizó una encuesta sobre prácticas y estilos de vida en ocio, administrada a jóvenes con discapacidad de 15 a 29 años, en una aproximación tanto objetiva (actividades, prácticas y consumos, tiempos, espacios y recursos) como subjetiva (motivaciones, intereses, opiniones, valores, preferencias, beneficios y barreras). Se consideró que, con un 95,5 \% de confianza y un $5 \%$ de error, el tamaño muestral debía ascender a 400 individuos. De cara a distribuir la muestra de un modo equitativo, se procedió a la estratificación de la misma en función de tipo de discapacidad, sexo y edad. Para recoger la oferta de ocio para jóvenes con discapacidad que se realiza la comunidad se elabora un cuestionario autoadministrado que se envió por correo electrónico a más de 650 agentes que trabajan en alguno de los ámbitos del ocio (cultura, turismo, deporte y recreación) en el sector público y privado con y sin ánimo de lucro y las áreas/departamentos de juventud.

\section{EXPERIENCIAS DE OCIO DE LOS JÓVENES CON DISCAPACIDAD}

Este apartado profundiza en las experiencias de ocio de los jóvenes con discapacidad. Para ello describe la caracterización de las prácticas de ocio, las demandas en ocio de los jóvenes con discapacidad y la configuración de los estilos de vida del colectivo estudiado. Diferentes autores han abordado el tema de la experiencia de ocio como factor de desarrollo humano desde distintas disciplinas (Csikszentmihalyi, 1997; Neulinger, 1980; Tinsley y Tinsley, 1986; Iso-Ahola, 1980; Elías y Dunning, 1988). Una experiencia es óptima (Csikszentmihalyi, 1997) cuando la persona percibe que quiere y debe hacer algo (percepción de desafío) y que es capaz de hacer (tiene habilidades). El interés de la experiencia de ocio no está tanto en el tipo de actividad, sino en los "desafíos" que proporciona a la persona y en el disfrute. Esta se articula con base en componentes personales (psicológicos y físicos) y sociales (más vinculados a diversas características del entorno). Según Iso-Ahola (1980), desde la perspectiva psicológica la experiencia de ocio se entiende como un estado mental subjetivo, resultado de la libre elección y llevada a cabo por razones intrínsecas que dependen de la persona que la experimenta. Lograr que la vivencia de ocio sea una experiencia valiosa implica cambios conceptuales, actitudinales y estructurales, y supone asumir (Cuenca, 2003), que frente a un ocio consumo, hay que trabajar un ocio experiencial. En este trabajo de investigación se entiende la experiencia de ocio como una realidad con vertiente objetiva (sujeto, grupo, actividad, tiempo, espacio y recursos) y vertiente subjetiva (beneficios, intereses y opiniones, motivaciones y valores).

\section{Práctica de actividades de ocio}

La juventud con discapacidad practica una media de 5,8 actividades distintas de ocio.

Atendiendo al tipo de actividades de ocio practicadas por la juventud con discapacidad, agrupadas estas por ámbitos de ocio, se observa cómo las actividades cul- 
turales, deportivas y recreativas son desarrolladas por más del $80 \%$ de la juventud; las actividades de carácter turístico son las de menor presencia. La práctica deportiva se mantiene en todas las franjas de edad, en mayor medida durante el periodo de 20-24 años. A mayor edad aumenta la participación en cultura. Las prácticas turísticas disminuyen según aumenta la edad. Al aumentar el nivel de estudios aumentan las prácticas culturales y deportivas. Las actividades culturales y deportivas aparecen más en jóvenes que viven en su propio hogar.

Gráfico 1. Volumen de práctica por ámbito de ocio

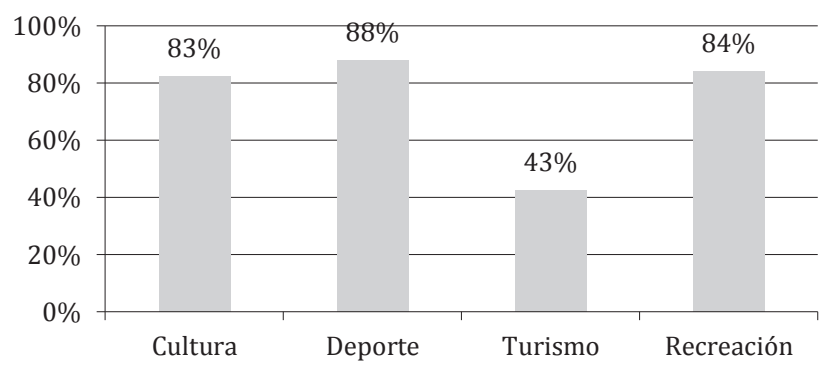

Fuente: Elaboración propia.

Atendiendo al tipo de actividades de ocio practicadas por la juventud con discapacidad, agrupadas estas por ámbitos de ocio, se observa cómo las actividades culturales, deportivas y recreativas son desarrolladas por más del $80 \%$ de la juventud; las actividades de carácter turístico son las de menor presencia. La práctica deportiva se mantiene en todas las franjas de edad, en mayor medida durante el periodo de 20-24 años. A mayor edad aumenta la participación en cultura. Las prácticas turísticas disminuyen según aumenta la edad. Al aumentar el nivel de estudios aumentan las prácticas culturales y deportivas. Las actividades culturales y deportivas aparecen más en jóvenes que viven en su propio hogar.

Ninguna actividad es practicada por más del $60 \%$ de jóvenes con discapacidad. En la tabla se presentan las actividades clasificadas en tres niveles de práctica, según el porcentaje de jóvenes que las practican. Así, estar con amigos, escuchar música y pasear son los tres tipos de actividades más extendidas entre el colectivo encuestado.
Tabla 1. Presencia de tipos de prácticas entre la juventud con discapacidad

\begin{tabular}{|c|c|c|}
\hline & Tipo de práctica & $\begin{array}{l}\text { \% n de } \\
\text { muestra }\end{array}$ \\
\hline \multirow{3}{*}{ 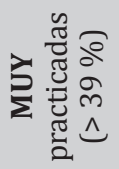 } & Salir o estar con amigos & $60 \%$ \\
\hline & Escuchar música & $45 \%$ \\
\hline & Pasear & $44 \%$ \\
\hline \multirow{8}{*}{ 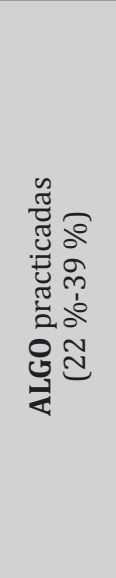 } & Salir de vacaciones & $38 \%$ \\
\hline & Navegar por Internet & $37 \%$ \\
\hline & Deporte individual & $32 \%$ \\
\hline & Ir al cine & $32 \%$ \\
\hline & Leer & $24 \%$ \\
\hline & Ir de copas o a discotecas & $24 \%$ \\
\hline & Deportes de equipo & $23 \%$ \\
\hline & $\begin{array}{l}\text { Ver en la TV programas } \\
\text { de entretenimiento }\end{array}$ & $23 \%$ \\
\hline \multirow{17}{*}{ 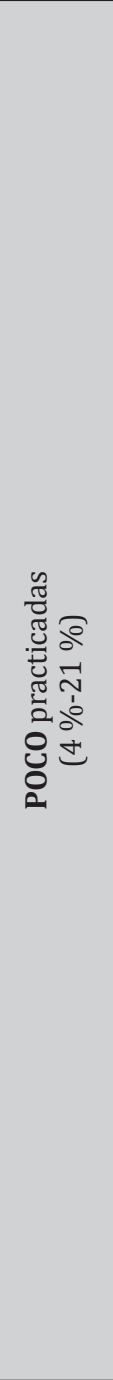 } & Ir de campamentos & $21 \%$ \\
\hline & Hacer excursiones & $19 \%$ \\
\hline & $\begin{array}{l}\text { Ver en la TV u oír en la radio } \\
\text { programas deportivos }\end{array}$ & $17 \%$ \\
\hline & $\begin{array}{l}\text { Hacer alguna actividad artística } \\
\text { (pintar...), musical (cantar...) }\end{array}$ & $17 \%$ \\
\hline & $\begin{array}{l}\text { Ir a fiestas, celebraciones } \\
\text { de amigos o familiares }\end{array}$ & $17 \%$ \\
\hline & Ir a conciertos & $16 \%$ \\
\hline & Videojuegos & $13 \%$ \\
\hline & Ir al campo, a la montaña o a la playa & $10 \%$ \\
\hline & $\begin{array}{l}\text { Ver en TV programas culturales } \\
\text { (documentales, debates...) }\end{array}$ & $10 \%$ \\
\hline & Ir a museos, exposiciones & $10 \%$ \\
\hline & Colaborar con mi asociación & $10 \%$ \\
\hline & Oír radio & $9 \%$ \\
\hline & Asistencia a espectáculos deportivos & $8 \%$ \\
\hline & $\begin{array}{l}\text { Hacer manualidades (bricolaje, } \\
\text { cerámica,...) }\end{array}$ & $5 \%$ \\
\hline & $\begin{array}{l}\text { Juegos de mesa (cartas, } \\
\text { dominó, parchís...) }\end{array}$ & $5 \%$ \\
\hline & $\begin{array}{l}\text { Hobbies (trenes, coches, } \\
\text { plantas, cocinar...) }\end{array}$ & $5 \%$ \\
\hline & Ir al teatro & $4 \%$ \\
\hline
\end{tabular}

Fuente: Elaboración propia. 
Gráfico 2. Prácticas según promotor de la actividad

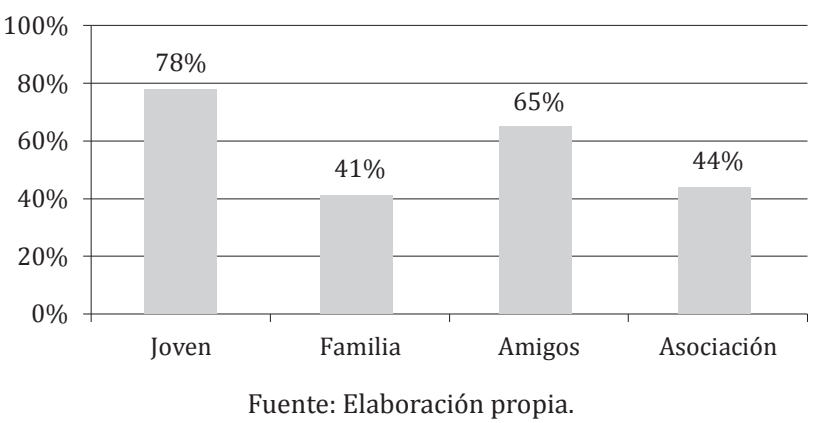

Un $78 \%$ de la juventud con discapacidad afirma que algunas de sus prácticas de ocio son organizadas por ellos mismos. Un $65 \%$ organiza también actividades junto con el grupo de amigos, un $44 \%$ afirma que algunas de sus actividades de ocio provienen del tejido asociativo y, por último, un $41 \%$ señala al núcleo familiar como promotor de sus prácticas.

Gráfico 3. Prácticas según compañía de la actividad

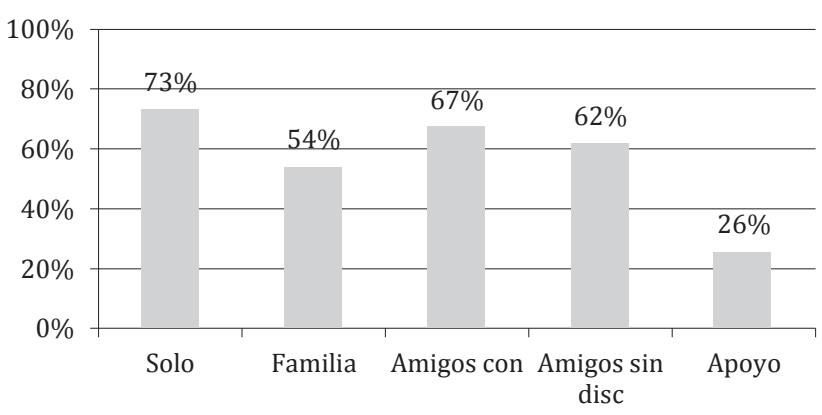

Fuente: Elaboración propia.

Un $73 \%$ de la juventud con discapacidad afirma que realiza actividades de ocio sin compañía, un $67 \%$ del colectivo disfruta de actividades junto con amigos/as con discapacidad, un $62 \%$ afirma que algunas de sus actividades de ocio las comparte con personas sin discapacidad, y un $54 \%$ señala además a la familia como compañeros. Compartir actividades de ocio con amigos sin discapacidad aumenta en paralelo al aumento de nivel educativo. En el grupo de jóvenes con discapacidad que vive en el hogar familiar es fundamental el papel del grupo de amigos. Los que viven con la familia hacen más actividades de ocio de manera individual o con amigos con o sin discapacidad.
Gráfico 4. Prácticas según frecuencia de actividad

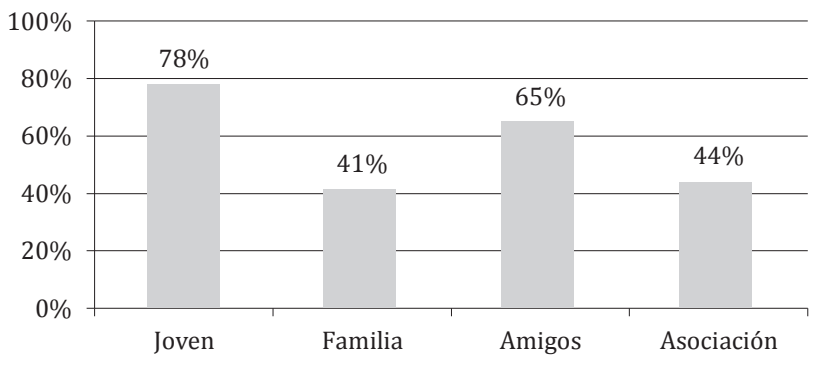

Fuente: Elaboración propia.

Atendiendo a la frecuencia de práctica de actividades de ocio, se observa cómo un 74 \% de la juventud con discapacidad practica alguna actividad de ocio al menos 2 o 3 días por semana. La periodicidad semanal está presente en el $59 \%$ en al menos una actividad de ocio. El nivel de instrucción no produce cambios en la periodicidad.

Gráfico 5. Prácticas según entorno de desarrollo

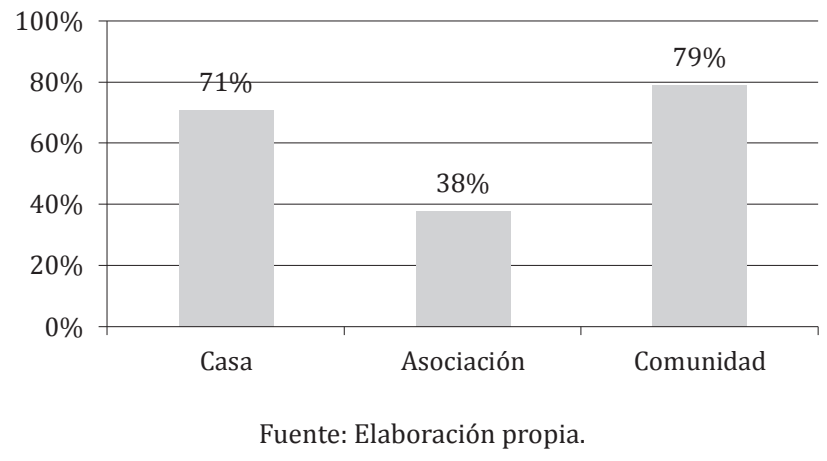

Al analizar el entorno de desarrollo de las actividades de ocio, un $79 \%$ de los jóvenes desarrollan alguna actividad de ocio en entornos comunitarios, o lo que es lo mismo, un $21 \%$ no lo hace y un $29 \%$ no practica actividades de ocio en su casa. Un $38 \%$ de la juventud con discapacidad señala la asociación como entorno de práctica. Y, a la inversa, un $62 \%$ del colectivo no identifica a los servicios de ocio de las asociaciones como entorno de ocio. 
Gráfico 6. Prácticas según nivel de satisfacción

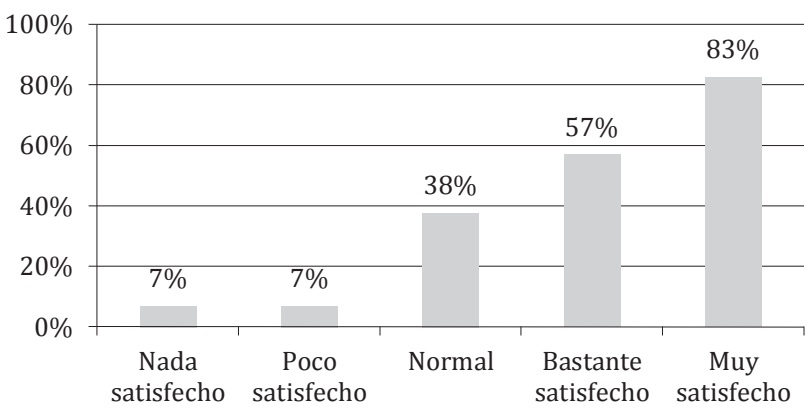

Fuente: Elaboración propia.

El 83 \% de los/las jóvenes con discapacidad afirman estar muy satisfechos con alguna de las actividades de ocio que practican. Tan solo un $7 \%$ dice no estar en absoluto satisfecho. En cuanto a la percepción de aburrimiento, tan solo un $8 \%$ afirma aburrirse en su tiempo libre.

Gráfico 7. Participación en servicios de ocio

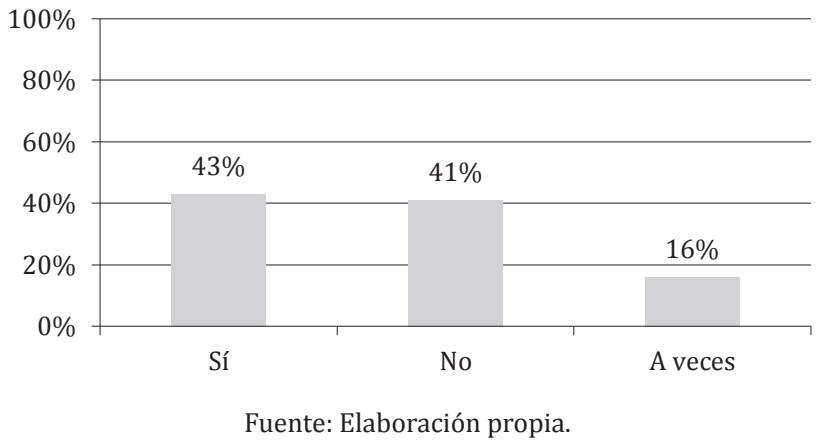

Como se observa en el gráfico, se ha obtenido prácticamente el mismo porcentaje de jóvenes con discapacidad que participan en servicios de ocio de asociaciones, que aquellos que no lo hacen. $\mathrm{Al}$ aumentar la edad disminuye la participación en el tejido asociativo.
Gráfico 8. Tipología de beneficios percibidos

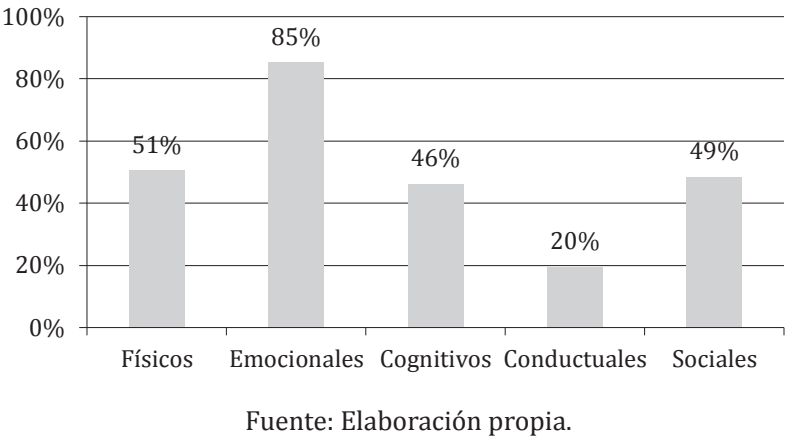

Prácticamente el total de la juventud con discapacidad afirma obtener algún tipo de beneficio tras la práctica de actividades de ocio. Así, el $85 \%$ de la juventud con discapacidad afirma obtener beneficios emocionales durante o tras sus prácticas de ocio, el resto de tipologías de beneficios ronda el $50 \%$ de casos con respuesta positiva.

\section{Demandas de prácticas de ocio entre la JcD3}

El $54 \%$ de la juventud encuestada afirma querer practicar nuevas actividades de ocio.

Ninguna actividad es demandada por más del $42 \%$ de la juventud con discapacidad. La práctica de deporte individual es la actividad más demandada entre el colectivo encuestado, y recordemos que también era una de las más extendidas como actividad habitual de ocio entre la juventud con discapacidad, practicada por el $32 \%$. De nuevo, una única actividad forma el bloque de prácticas con relativa presencia entre las demandas por el colectivo encuestado, ir al teatro. Destaca que ningún joven ha señalado el colaborar con el tejido asociativo entre el grupo de actividades de ocio que le gustaría desarrollar. Comparando los datos sobre actividad de ocio real y actividad de ocio deseada, se observa cómo las actividades que destacan por el porcentaje de jóvenes que manifiestan su deseo de práctica, son practicadas por un número reducido de jóvenes con discapacidad, y a la inversa ocurre lo mismo, aquellas actividades más practicadas, presentan índices bajos de demanda. Por sexo, las diferencias no son muy altas, pero se observa que los hombres demandan más actividades deportivas y recreativas y las mujeres aquellas prácticas relacio-

3 JcD: Juventud con Discapacidad. 
nadas con actividades culturales. A mayor edad menos demanda deportiva. En cuanto al resto de prácticas no se percibe ningún patrón claro.

Tabla 2. Tipos de prácticas demandadas entre la juventud con discapacidad

\begin{tabular}{|c|c|c|}
\hline Nivel de deseo & Tipo de práctica & $\begin{array}{c}\% \text { de deseo } \\
\text { de n de grupo }\end{array}$ \\
\hline Muy deseadas & Deporte individual & $42 \%$ \\
\hline $\begin{array}{l}\text { Bastante } \\
\text { deseadas }\end{array}$ & Ir al teatro & $19 \%$ \\
\hline \multirow{13}{*}{ Poco deseadas } & Deportes de equipo & $14 \%$ \\
\hline & Ir al cine & $12 \%$ \\
\hline & Ir a conciertos & $10 \%$ \\
\hline & Salir de vacaciones & $10 \%$ \\
\hline & $\begin{array}{l}\text { Ver en TV programas } \\
\text { culturales (documen- } \\
\text { tales, debates...) }\end{array}$ & $9 \%$ \\
\hline & $\begin{array}{l}\text { Asistencia a espec- } \\
\text { táculos deportivos }\end{array}$ & $8 \%$ \\
\hline & $\begin{array}{l}\text { Hacer alguna actividad } \\
\text { artística (pintar...), } \\
\text { musical (cantar...) }\end{array}$ & $8 \%$ \\
\hline & $\begin{array}{l}\text { Ir a museos, } \\
\text { exposiciones }\end{array}$ & $7 \%$ \\
\hline & $\begin{array}{l}\text { Ir al campo, a la mon- } \\
\text { taña o a la playa }\end{array}$ & $7 \%$ \\
\hline & $\begin{array}{l}\text { Hacer manualidades } \\
\text { (bricolaje, cerámica,...) }\end{array}$ & $6 \%$ \\
\hline & Videojuegos & $6 \%$ \\
\hline & Hacer excursiones & $5 \%$ \\
\hline & Ir de campamentos & $5 \%$ \\
\hline No demandadas & $\begin{array}{l}\text { Colaborar con mi } \\
\text { asociación }\end{array}$ & $0 \%$ \\
\hline
\end{tabular}

Fuente: Elaboración propia.

Si se analizan las barreras agrupadas según sean externas o internas, se observa cómo las internas tienen mayor peso e importancia para la juventud con discapacidad encuestada.

La propia discapacidad es la barrera señalada en mayor número de ocasiones como explicación ante la no práctica de actividades de ocio, a continuación se sitúa la falta de tiempo, junto con la falta de comunicación.
Gráfico 9. Barreras externas

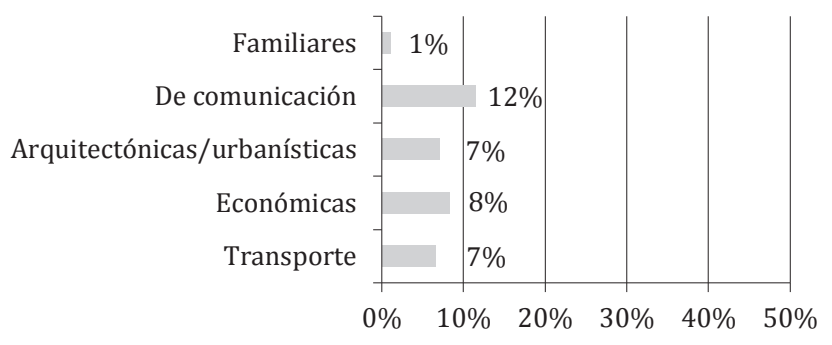

Fuente: Elaboración propia.

Gráfico 10. Barreras internas

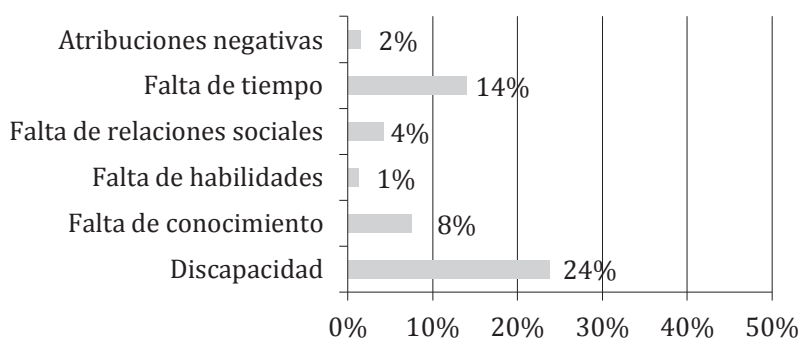

Fuente: Elaboración propia.

\section{Estilos de vida en ocio de la juventud con discapacidad}

Opiniones sobre ocio. Es importante destacar cómo la juventud en su conjunto valora en gran medida que las actividades de ocio son un elemento importante en sus vidas y es un espacio o ámbito vital adecuado para fraguar nuevas amistades. Por tanto, en esta línea, consideran que el ocio no es una pérdida de tiempo o un modo de tirar el dinero. Si agrupamos las opiniones en función de los factores subyacentes, se revela la existencia de seis opiniones generales diferentes sobre el ocio, de las cuales una de ellas tiene un carácter negativo. Se entiende el ocio como socialización, solidaridad, descanso, desarrollo personal o recreación.

Preferencias sobre ocio. Si se agrupan las respuestas aparecen dos grandes factores subyacentes a las preferencias. Por un lado, está la juventud con discapacidad que prefiere las emociones y la novedad en su experiencia de ocio frente a la tranquilidad y la seguridad en dichas actividades. Por otro lado, está el grupo de jóvenes con discapacidad que prefiere la autenticidad y la independencia, frente a la calidad de la actividad de ocio. A nivel general, es más habitual que la juventud con discapacidad prefiera desarrollar en su ocio vivencias cargadas de emociones y novedades que llenas de tranquilidad y seguridad. 
Motivaciones al ocio. Si agrupamos las motivaciones en sus factores subyacentes se revela que existen tres tipos de motivaciones que pueden impulsar o animar la práctica de actividades de ocio. Así, el ocio puede ser un espacio y un tiempo que posibilita el entrar en contacto con otras personas, el ocio como un tiempo durante el cual uno mismo espera desarrollarse como individuo, y por último un tiempo para descansar. En el gráfico se observa cómo el factor de socialización cobra mayor importancia en el colectivo de jóvenes con discapacidad, pero siendo los tres factores motivacionales importantes para el colectivo.

Valores. Destacar cómo la juventud en su conjunto afirma dotar de importancia a diversos valores de carácter vital. Al agrupar los valores en los componentes que lo forman, es posible extraer dos grandes factores que pueden determinar en mayor o menor medida la vivencia y experimentación individual de la práctica de actividades de ocio.

\section{EL ACCESO AL OCIO COMUNITARIO DE LOS JOVENES CON DISCAPACIDAD}

La programación de ocio comunitario se divide en dos apartados: análisis de la programación para jóvenes y la programación específica para jóvenes con discapacidad. En el primer apartado se ahonda en la presencia de jóvenes con discapacidad en la oferta comunitaria de ocio para población joven, y en el segundo se analizan de forma pormenorizada los programas de ocio que ofertados desde el entorno de ocio comunitario, su principal destinatario es el joven con discapacidad.

Presencia de JcD en la programación de ocio para jóvenes

Gráfico 11. Consideración de la discapacidad en la oferta

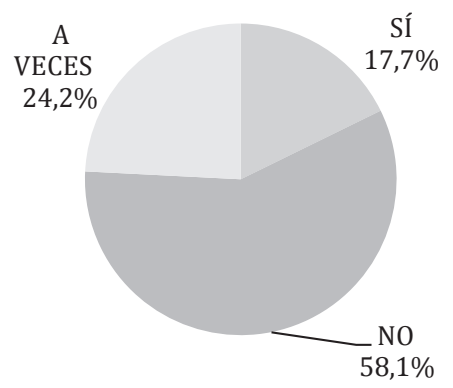

Fuente: Elaboración propia.
Se puede observar que en el 58,1 \% de los casos no se tiene en cuenta a la hora de diseñar y desarrollar la oferta de servicios y/o programas al colectivo de jóvenes con discapacidad. En cambio, el dato de "a veces" es similar y por lo tanto solamente en el $17,7 \%$ de los casos, a la hora de diseñar la oferta se tiene en cuenta al colectivo.

Gráfico 12. Experiencia de participación de la JcD

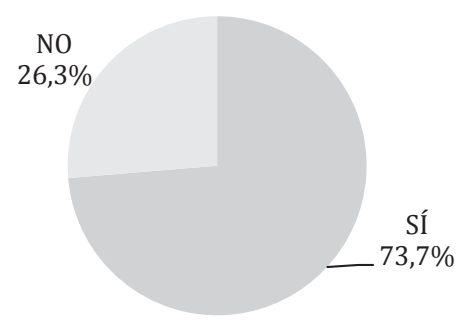

Fuente: Elaboración propia.

Con relación a la experiencia de participación de juventud con discapacidad en la oferta de servicios y programas de ocio se constata que es bastante amplia ya que casi el $74 \%$ de los profesionales afirma que han participado jóvenes con discapacidad.

Los servicios y programas de ocio que se organizan teniendo en consideración que puedan participar jóvenes con diferentes discapacidades son la realización de visitas guiadas adaptadas, la oferta de actividades deportivas en las que pueden participar, la oferta de actividades deportivas adaptadas a grupos concretos de jóvenes con discapacidad, la realización de actividades recreativas en lugares accesibles, sobre todo sin presencia de barreras arquitectónicas, y los servicios que prestan información sobre equipamientos y actividades de ocio que abordan cuestiones relacionadas con la accesibilidad a los mismos.

Aparecen en menor medida que las anteriores, ya que se citan dos veces o menos, pero que consideran a la juventud con discapacidad los siguientes programas: actividades de verano, monitores de apoyo, programas de ocio escolares, talleres, actividades de ocio específicas, actividades festivas, actividades recreativas adaptadas, intérprete de lengua de signos, y venta de entradas exclusivas y espacios reservados en las salas. 
Gráfico 13. Experiencia de participación de JcD

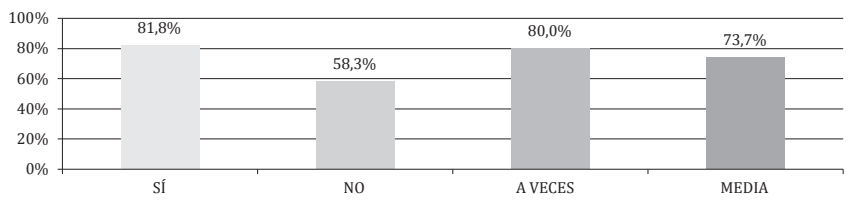

Fuente: Elaboración propia.

Teniendo en cuenta que hay agentes que programan y diseñan su oferta sin considerar al colectivo objeto de análisis, hay que destacar que cuando se tiene en cuenta, su participación es mayor, cercana al 80 \%. En cambio, en los casos en los que no se les considera, este porcentaje desciende hasta el $58 \%$.

Gráfico 14. Experiencia de participación según tipo de discapacidad

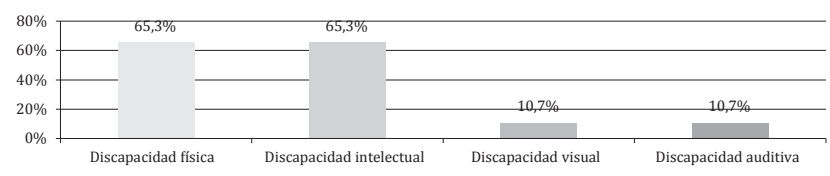

Fuente: Elaboración propia.
En el caso de que participen, lo más habitual es que participen en mayor medida los grupos de discapacidad física e intelectual, mientras que la presencia de los colectivos de discapacidad visual y auditiva está mucho menos presente.

En cuanto a la edad, se puede constatar de una presencia mayor de la población más joven, entre 15 y 19 años, un pequeño descenso de presencia en el colectivo entre 20 y 24 años y un pequeño aumento en la última franja de edad que discurre entre 25 y 29 años.

Gráfico 15. Rango de edad de los participantes

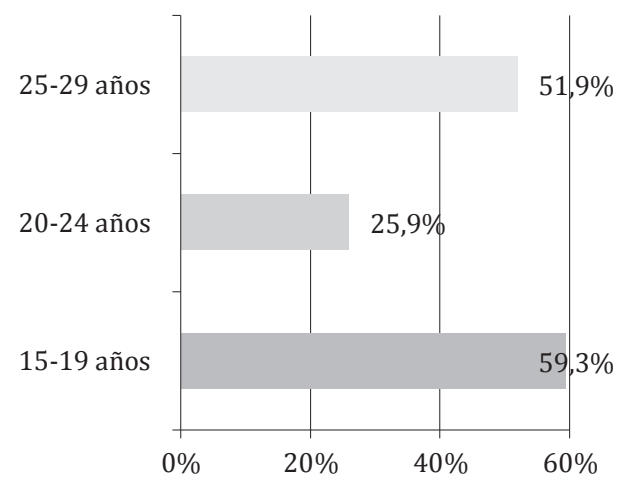

Fuente: Elaboración propia.

Gráfico 16. Compañía en la práctica

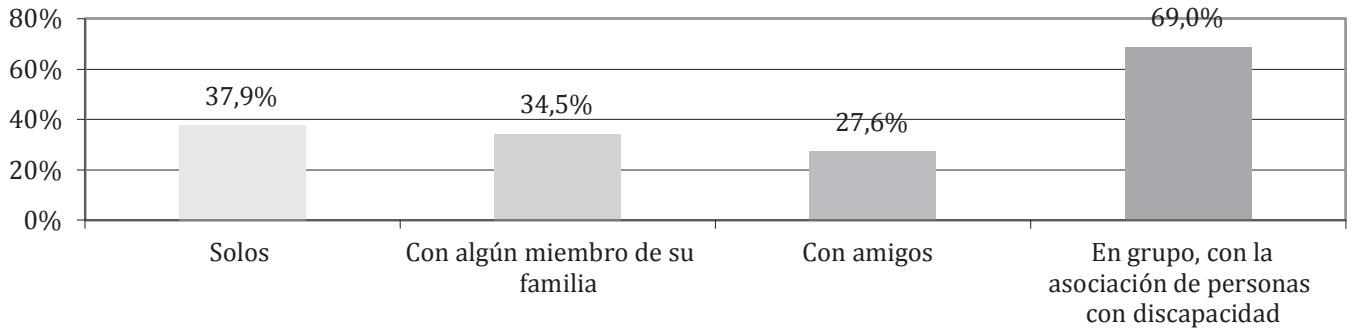

Fuente: Elaboración propia.

Cuando se analiza el contexto social de la juventud con discapacidad en cuanto a su participación en actividades de ocio, se descubre que siguen acudiendo a las actividades principalmente en grupo, con la asociación de discapacidad, y en segundo lugar solos o con algún miembro de su familia. La situación más inusual es que acudan con sus amistades.
Gráfico 17. Número de jóvenes con discapacidad participantes

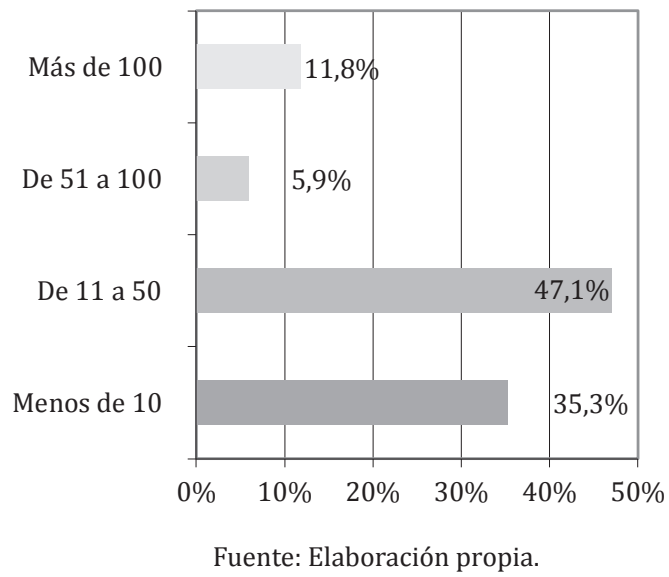


El número de participantes sigue siendo bastante bajo y lo más común es que participen menos de 50 jóvenes con discapacidad en las actividades de ocio ofertadas a la juventud.

\section{Programación de ocio comunitaria para JcD}

Gráfico 18. Consideración discapacidad en la oferta

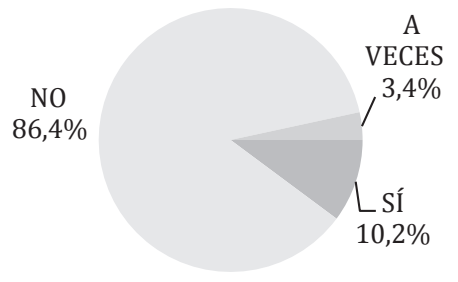

Fuente: Elaboración propia.

Gráfico 19. Experiencia de participación según tipo de discapacidad

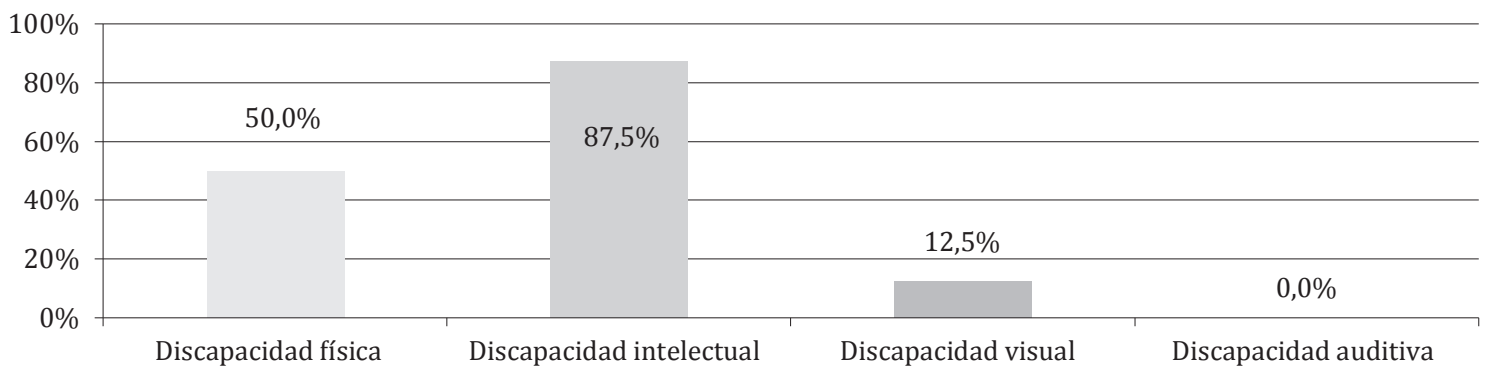

Fuente: Elaboración propia.

En el caso en el que los agentes programen de forma específica para el colectivo de jóvenes con discapacidad, lo más habitual es que lo hagan teniendo en cuenta la discapacidad intelectual en un 87,5\%. En la mitad de los casos también tienen en cuenta la discapacidad física, pero en el caso de la visual el porcentaje desciende hasta el $12,5 \%$ y la discapacidad auditiva no tiene presencia.

En cuanto al rango de edad, como ocurría en la programación para jóvenes, sigue siendo lo más habitual los extremos de edad, es decir que participen los más jóvenes y los más mayores.
El 86,4 \% de los agentes no diseña ningún tipo de servicio o programa específico dirigido a jóvenes con discapacidad. Solamente el $10 \%$ de los agentes realizan este tipo de programación de forma habitual y un 3,4 \% lo hace de forma ocasional.

La oferta de programas de ocio para jóvenes con discapacidad en equipamientos comunitarios se centra sobre todo en organizar cursos y talleres, ofertar actividades deportivas, y adaptar visitas guiadas a grupos específicos. Las exposiciones adaptadas, atención individualizada y oferta deportiva adaptada aparecen en menor medida. 
La compañía con la que realizan estas prácticas de ocio se ve influenciada por la especificidad de las actividades, por lo que en todos los casos la realizan en grupo, con miembros de la misma asociación de discapacidad. Ocasionalmente con algún familiar.

El número de participantes sigue siendo pequeño y quizá coincida con la presencia de jóvenes con discapacidad en las programaciones para jóvenes: normalmente inferior a 50 jóvenes.

Gráfico 22. Número de JcD participantes

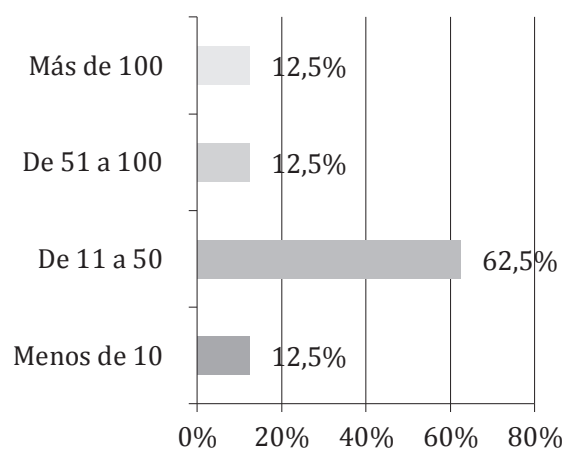

Fuente: Elaboración propia.

\section{DISCUSIÓN DE RESULTADOS Y CONCLUSIONES}

La juventud con discapacidad (JcD), al igual que el resto de la población joven, valora el ocio como elemento importante en sus vidas, como un espacio vital adecuado para fraguar nuevas amistades, y como ámbito de desarrollo personal, al igual que el conjunto de la población joven sin discapacidad. Así lo muestran los datos del sondeo de opinión realizado por el Instituto de la Juventud (INJUVE) en 2014, sobre "Jóvenes valores y ciudadanía”, en el que el ocio es identificado como uno de los aspectos importantes en la vida personal, situado por delante de otros valores como la política, ganar dinero, la religión o la sexualidad. La JcD en su conjunto manifiesta sentir diversas motivaciones que le impulsan a la práctica de actividades de ocio, destaca la valoración positiva que hacen del hecho de compartir el tiempo con otras personas, así como la motivación hacia el aprendizaje de cosas nuevas. Los jóvenes prefieren vivencias de ocio emocionantes y novedosas (Nimrod, Megan y Kleiber, 2016).
El colectivo presenta un patrón homogéneo en cuanto a volumen y diversidad de actividades de ocio se refiere. La gran mayoría de la JcD (8 de cada 10) practica tanto actividades de carácter cultural como deportivas y recreativas. La oferta de actividades de ocio dirigidas a la juventud, o específicamente al colectivo con discapacidad, es múltiple y variada, por tanto la atomización de la práctica por tipologías es un hecho. Con el aumento de la edad se identifica una transformación en la tipología de prácticas desarrolladas, hecho no exclusivo de este colectivo, sino generalizado entre la población joven (INJUVE, 2010; 2015a).

Es del todo relevante el papel de la JcD en la organización de sus actividades de ocio y, además, el papel del grupo de amigos. Otros actores, como son la familia y el tejido asociativo, susceptibles de ser promotores en la organización del ocio del colectivo, van disminuyendo su presencia a medida que el/la joven con discapacidad va creciendo y aumenta su autonomía (Ortega, Lazcano, Manuel, 2015), y por tanto su capacidad en la toma de decisión, transformación propia de transitar por la juventud, del proceso de maduración y crecimiento personal.

El entorno de mayor referencia para la JcD como espacios clave en el desarrollo de sus actividades de ocio, es el comunitario, al igual que el resto de la juventud (INJUVE, 2015b; Ortega, Lazcano, Manuel, 2015). La JcD desempleada realiza más actividades de ocio en casa, que los estudiantes y los ocupados, estos dos grupos utilizan como primer entorno de ocio la comunidad. Sobre la compañía, es posible concluir que el volumen de actividades de ocio que los jóvenes con discapacidad desarrollan en compañía de una persona de apoyo o con otros jóvenes con discapacidad disminuye cuando él o la joven presentan niveles de estudios más elevados, lo que indica la posibilidad de mayor autonomía o presencia de discapacidades que conllevan funcionamiento independiente. Por tanto, el hecho de compartir actividades de ocio con amigos sin discapacidad aumenta en paralelo con el aumento del nivel educativo.

La mitad de la JcD demanda nuevas actividades de ocio a incorporar a su repertorio de prácticas habituales. A mayor edad y nivel de instrucción, mayor deseo de practicar nuevas actividades de ocio. La principal demanda se centra en el ámbito del deporte, actividad por otro lado de las más extendidas como actividad habitual de ocio. La propia discapacidad es el tipo de barrera que 
en mayor medida retrae la práctica de actividades de ocio entre la juventud, principalmente en edades más tempranas, hecho que se externaliza cuando la discapacidad es asumida con la maduración del joven, de la misma manera, el nivel educativo de la propia juventud con discapacidad favorece la disminución de la importancia otorgada a la propia discapacidad. El tema de barreras ha sido ampliamente estudiado en el colectivo de discapacidad por la incidencia que tiene en la no realización de actividades de ocio (Jackson, 2000; Mahy, Shields, Taylor y Dodd, 2010; Leadbetter y O'Connor, 2013.). Al igual que el resto de la juventud, el colectivo con discapacidad, no manifiesta ningún interés en la colaboración en su tiempo libre con el tejido asociativo.

La JcD manifiesta estar satisfecha con su tiempo de ocio y opina que este es un momento para el descanso y la socialización, a la par que un espacio vital para el desarrollo personal. Esta correlación entre satisfacción con el ocio y el desarrollo personal ha sido ampliamente demostrada a través de investigaciones empíricas en el campo de los estudios de ocio (McGuire, Dottavio y O'Leary, 1987; Badia y Araujo, 2009; Benson, Scales, Hamilton y Sesma, 2010; Badia, Orgaz, Verdugo y Ullán, 2013). Así, la motivación hacia la práctica de actividades de ocio tiene su origen para este colectivo en la posibilidad de desarrollar nuevas competencias, cuyos principales beneficios percibidos son los de carácter emocional.

Con relación a la atención a la discapacidad en la programación de ocio para jóvenes, cabe decir que no se contempla al colectivo de jóvenes con discapacidad. El tener experiencias de participación de JcD facilita contemplar a este colectivo en la programación tal y como apuntan estudios sobre la situación de las personas con discapacidad (Comité Español de Representantes de Personas con Discapacidad, CERMI 2011 a y b; 2012). Los servicios y programas de ocio que se organizan teniendo en consideración a JcD son visitas guiadas adaptadas, actividades deportivas, actividades recreativas accesibles, y que los servicios de información incluyen cuestiones sobre accesibilidad. En el caso de la programación para jóvenes, se da más presencia de las discapacidades física e intelectual (Confederación Española de Organizaciones en favor de las Personas con Discapacidad Intelectual o del Desarrollo, FEAPS, 2002). Destaca de la programación específica para jóvenes con discapacidad que la mayoría de los agentes no diseñan ningún tipo de servicio o programa dirigido a JcD. La oferta de programas de ocio para JcD en la oferta comunitaria se centra en organizar cursos y talleres, actividades deportivas adaptadas, y adaptar visitas guiadas a grupos. La programación específica para JcD contempla principalmente la discapacidad intelectual (FEAPS, 1999) y en menor medida la discapacidad física, seguida de la discapacidad visual y la ausencia de la auditiva. Los JcD acuden en grupo con la asociación de personas con discapacidad a las actividades de ocio específicas para el colectivo, realidad que ya se recoge en estudios previos en los que se denota la segmentación del sector (Madariaga, 2011).

Las condiciones de inclusión física y comunicativa en los equipamientos y servicios de ocio (Gorbeña, Madariaga y Rodríguez, 2002) se caracterizan porque en la mayoría de los casos los equipamientos cumplen con todas las medidas de accesibilidad que marca la legislación vigente. En cuanto a la inclusión física en equipamientos y servicios de ocio, la mayoría disponen de rampas, ascensor o aseos adaptados. En menor medida se constata la reserva de espacio o una buena movilidad interior. Muchos de los equipamientos disponen de la información al alcance visual y más de la mitad permite el alcance manual, y usa formatos accesibles. La señalización cumple las condiciones de accesibilidad. El contenido y los soportes diversos no están tan generalizados. Con relación a la inclusión social en los servicios y programas de ocio (Madariaga, Lazcano, Doistua y Lázaro, 2012), las principales soluciones que se adoptan son informar de la oferta accesible al usuario, proporcionar monitores de apoyo a la actividad, brindar ayuda personal cuando se solicita, desarrollar planes de accesibilidad en el equipamiento, instalar sillas elevadoras, elaborar videos descriptivos sobre partes inaccesibles, y adaptar la metodología. En cuanto a las opiniones del personal (Dattilo, 2004), la mayoría afirma reconocer el derecho al ocio y valora como importante la accesibilidad. Muchos consideran conveniente que los jóvenes con discapacidad acudan acompañados de monitores propios. Entre las medidas de acción positiva, las más comunes, aunque en ningún caso superan la mitad, son el incluir a la JcD entre los públicos destinatarios, la gratuidad del acompañante y distribuir la información entre las asociaciones de discapacidad.

Por último, cabe señalar las necesidades para la inclusión de los jóvenes con discapacidad en la oferta de ocio comunitaria (Organización de Naciones Unidas, ONU, 
2006). Las cuestiones fundamentales que se identifican desde la oferta comunitaria de ocio son mejorar la accesibilidad del acceso y la comunicación en equipamientos de ocio, ofertar actividades adaptadas, aumentar la formación de profesionales, desarrollar programaciones específicas, consolidar una política inclusión y no discriminación, generar acciones divulgativas, aumentar la oferta de ocio adaptada, conocer las necesidades de los participantes, fomentar la autonomía personal, coordinarse con asociaciones del ámbito de la discapacidad, posibilitar la participación activa, y proporcionar desde las asociaciones monitores propios de apoyo para la actividad.

En conclusión, se ha avanzado mucho en el acceso y la participación de los jóvenes con discapacidad a la oferta comunitaria de ocio (Madariaga, 2011). Por un lado, los profesionales cada vez tienen más experiencias con jóvenes con discapacidad, hecho que actúa como un factor determinante para programar de forma más incluyente. Pero, por otro lado, las condiciones de inclusión física y comunicativa pese a haber mejorado no cubren todas las necesidades del colectivo joven con discapacidad, y en materia de inclusión social hay que continuar trabajando por la cultura de la inclusión y por incorporar más elementos que faciliten la plena participación, la equiparación de oportunidades (LIONDAU, 2003) y el poder ejercer el derecho al ocio (Lázaro, Madariaga, Lazcano y Doistua, 2012).

\section{REFERENCIAS}

Badia, M., y Araújo, E. L. (2009). El ocio en las personas con discapacidad intelectual: participación y calidad de vida a través de las actividades de ocio. Siglo Cero, 40 (3), 231, 30-44.

Badia, M., Orgaz, M. B., Verdugo, Á., y Ullán, A. M. (2013). Patterns and determinants of leisure participation of youth and adults with developmental disabilities. Journal of Intellectual Disability Research, 57, 4, 319-332.

Benson, P. L., Scales, P. C., Hamilton, S. F., y Sesma, A. (2007). Positive youth development: theory, research, and applications. En Damon, W. y Lerner, R. . (Ed.), Handbook of Child Psychology (pp. 894-941). New Jersey John Wiley \& Sons.

Cátedra Ocio y Discapacidad (2014). Manifiesto por un ocio inclusivo. Bilbao: Instituto de Estudios de Ocio.
Comité Español de Representantes de Personas con Discapacidad (CERMI) (ed.) (2011a). Estrategia de acción pública en materia de cultura inclusiva (Colección Telefónica Accesible, 13). Madrid: CERMi y Cinca.

Comité Español de Representantes de Personas con Discapacidad (CERMI) (ed.) (2011b). Modelo de plan de acción local para la inclusión de las personas con discapacidad 2012-2015. Colección Barclays Diversidad e Inclusión. Madrid: Cinca.

Comité Español de Representantes de Personas con Discapacidad (CERMI) (ed.) (2012). El sector de la discapacidad: realidad, necesidades y retos futuros. Análisis de la situación de la población con discapacidad $y$ de las entidades del movimiento asociativo $y$ aproximación a sus retos y necesidades en el horizonte de 2020 (59). Madrid: Cinca.

Confederación Española de Organizaciones en Favor de las Personas con Discapacidad Intelectual (FEAPS) (ed.) (2002). Estudio de realidad de los servicios de ocio. Madrid: FEAPS.

Confederación Española de Organizaciones en Favor de las Personas con Discapacidad Intelectual (FEAPS) (ed.) (1999). Ocio en comunidad. Orientaciones para la calidad. Manual de buena práctica. Madrid: FEAPS.

Csiksentmihalyi, M. (1997). Fluir: Una psicología de la felicidad. Barcelona: Kairós.

Cuenca, M. (2000). Ocio humanista. Documentos de estudios de ocio, 16. Bilbao: Universidad de Deusto.

Cuenca, M. (2003). El ocio como experiencia de desarrollo humano, un reto para el siglo XxI. En C. de la Cruz (ed.), Los retos del ocio y la discapacidad en el siglo XXI (pp. 33-66). Bilbao: Universidad de Deusto.

Dattilo, J. (2004). Servicios de ocio inclusivo. En Y. Lázaro (ed.), Ocio, inclusión y discapacidad. Documentos de estudios de ocio, 28 (pp. 21-50). Bilbao: Universidad Deusto.

Doistua, J., Lazcano, I., y Madariaga, A. (2011). Estrategia de inclusión en ocio para la juventud con discapacidad. En A. Madariaga y J. Cuenca (eds.), Los valores del ocio. Documentos de estudios de ocio, 43 (pp. 279-292). Bilbao: Universidad de Deusto.

Elías, N., y Dunning, E. (Eds.) (1988). Deporte y ocio en el proceso de civilización. México: Fondo de Cultura Económica.

Gorbeña, S., Madariaga, A., y Rodríguez, M. (2002). Protocolo de evaluación de las condiciones de inclusión en equipamientos de ocio. Documentos de estudios de ocio, 22. Bilbao: Universidad de Deusto. 
Instituto de la Juventud (INJUVE) (2010). Juventud en cifras: ocio y tiempo libre. Madrid: Observatorio Juventud en España

Instituto de la Juventud INJUve) (2014). Jóvenes, satisfacción personal, participación asociativa y voluntariado. Madrid: Observatorio Juventud en España.

Instituto de la Juventud (INJUVE) (2015a). Sondeo de opinión: jóvenes, valores y ciudadanía. Madrid: Observatorio Juventud en España.

Instituto de la Juventud (INJUVE) (2015b). Jóvenes, ocio y consumo. Madrid: Observatorio Juventud en España.

Iso-Ahola, S. E. (1980). The social psychology of leisure and recreation. Dubuque: W. C. Brown.

Jackson, E. L. (2000). Will research on leisure constraints still be relevant in the twenty-first century?. Journal of Leisure Research, 32(1), 62-68.

Lázaro, Y., Madariaga, A., Lazcano, I., y Doistua, J. (2012). El derecho al ocio: un derecho humano en ocasiones desconocido. Siglo Cero, 43(1), (formato CD). núm. 241.

Lazcano, I., Doistua, J., Lázaro, Y., y Madariaga, A. (2012). La experiencia de ocio como factor de desarrollo humano en el colectivo de personas con discapacidad. Revista Siglo Cero, 43(1), (formato CD). núm. 241.

Lazcano, I., Madariaga, A., Doistua, J., Y Cuenca, J. (2012). Active aging and its incidence in the leisure experience. Journal of Aging and Physical Activity, 20, 62-63.

Leadbetter, C., y O'Connor, N. (2013). Healthy attendance? The impact of cultural engagement and sports participation on health and satisfaction with life in Scotland. Scotland: Scottish Government Social Research. Disponible en: http://www.scotland.gov. uk/Resource/0043/00430649.pdf

LIONDAU (BOE 289/2003). Ley 51/2003, de 2 de diciembre, de igualdad de oportunidades, no discriminación y accesibilidad universal de las personas con discapacidad.
Madariaga, A. (2011). Los servicios de ocio de las asociaciones de discapacidad. Cuadernos de estudios de ocio, 13. Bilbao: Universidad de Deusto.

Madariaga, A., y Lazcano, I. (2014). Estudio sobre la situación del ocio de la juventud con discapacidad en Bizkaia. Bilbao: Diputación Foral de Bizkaia.

Madariaga, A., Lazcano, I., Doistua, J., y Lázaro, Y. (2012). La inclusión en ocio: ¿Un elemento de innovación en la sociedad actual? ¿Cómo se puede avanzar? Revista Siglo Cero, 43(1), (formato CD). núm. 241.

Madariaga, A., Lazcano, I., y Doistua, J. (2010). El ocio de la juventud con discapacidad en Euskadi. Estudios de ocio. Bilbao: Universidad de Deusto.

Mahy, J., Shields, N., Taylor, N. y Dodd, K. (2010). Identifying facilitators and barriers to physical activity for adults with Down syndrome. Journal of Intellectual Disability Research 54, 795-805.

McGuire, M., Dottavio, F., y O'Leary, J. (1987). The relationship of early life experiences to later life leisure envolvement. Leisure Sciences, 9, 251-257.

Organización de Naciones Unidas (ONU) (2006). Convención internacional de los derechos de las personas con discapacidad. Informe. A/AC.265/2006/4. Disponible en http://www.dpi.org

Neulinger, J. (1980). The psychology of leisure. Springfield: Charles C. Thomas.

Nimrod, G., Megan, J., y Kleiber, D. A. (2016). Leisure and aging qualitative research. 15 years into the third millennium. Journal of Leisure Research, 48(1), 12-14.

Ortega, C., Lazcano, Manuel, M. (2015). Espacios de ocio para jóvenes, de la monitorización a la autogestión. Pedagogía Social. Revista Interuniversitaria, 25, 69-8. doi:10.7179/PSRI_2015.25.4.

San Salvador del Valle, R., y Lazcano, I. (2003). Equipamientos municipales de proximidad. Gijón: Ediciones Trea.

Tinsley, H. E. A., Tinsley, D. J. (1986). A theory of atributes, benefits and causes of leisure experience. Leisure Sciences, 8(1), 1-45. 
